\title{
Temperature Dependence of Electrical and Thermal Resistivities of Noble Metals
}

\author{
B. S. Semwal, P. K. Sharma, and K. N. Mehrotra \\ Physics Department, University of Allahabad, Allahabad, India
}

(Z. Naturforsch. 26 a, 735-738 [1971] ; received 25 August 1970)

\begin{abstract}
The electrical and thermal resistivities of noble metals copper, silver and gold have been determined at different temperatures in the free electron approximation using Sharma and Joshi's model for the phonon dispersion relations. A modified Houston's method is used to evaluate the formidable integral over the scattering vector. The normal and Umklapp contributions to the resistivity are considered separately from the conservation laws of wave vector. The theoretical and experimental resistivity curves are found to be of similar nature, but the agreement is not detailed. The Lorentz numbers of noble metals deduced from theoretical and experimental resistivity data show considerable deviation from Wiedemann-Franz law.
\end{abstract}

\section{Introduction}

In pure metals, the resistance to the flow of electricity and heat is mainly due to the scattering of conduction electrons by the thermal vibrations of ions. The crux of the problem for the calculation of electrical and thermal resistivities due to phonon scattering lies in the determination of the phonon spectrum of normal modes and the matrix element of electron-ion core scattering ${ }^{1}$. During the last few years, a number of models ${ }^{2}$ have been worked out for studying the phonon dispersion relations in metals by making specific use of conduction electrons. One of the authors (P.K.S.) and JosHI ${ }^{3,4}$ have proposed a successful theory of this type by visualising a metallic crystal as a quasi-infinite lattice of bare ions embedded in a sea of electrons. The volume forces due to the compressibility of the electron gas and its interaction with ions are obtained from an average over a Wigner-Seitz sphere. The model has been shown to provide a satisfactory description of the phonon spectrum and the thermal properties of a number of metals ${ }^{5}$.

In this paper, we present a computation of electrical and thermal resistivities of the noble metals copper, silver and gold within the free electron approximation using Sharma and Joshi's model for the phonon dispersion relations. The normal and Umklapp contributions are considered separately

1 J. M. Ziman, Electrons and Phonons, Oxford University Press, New York 1962, Chap. VII and IX.

2 J. De Launay, Solid State Physics, Academic Press, Inc., New York 1956, Vol. 2, p. 220.

3 P. K. Sharma and S. K. Joshi, J. Chem. Phys. 39, 2633 [1963].

${ }^{4}$ P. K. Sharma and S. K. Joshi, J. Chem. Phys. 40, 662 [1964]. from the conservation laws of wave vectors and the geometry of reciprocal space. The calculated resistivities are used to deduce the Lorentz numbers of these metals.

\section{Theory}

ZIMAN $^{1}$ and others ${ }^{6,7}$ have given general procedures for evaluating the transport coefficients of metals using a variational principle. For lattices with cubic symmetry, the expressions for electrical resistivity $\varrho$ and thermal resistivity $W$ due to phonon scattering in the first Born approximation considering the simplest trial function are given by

$$
\varrho=\frac{3 \pi \hbar}{2 e^{2} M N k_{\mathrm{B}} T k_{\mathrm{F}}^{2} S_{\mathrm{F}}^{2}}
$$

$\cdot \sum_{p} \iint \frac{K^{2}\left(\boldsymbol{K} \cdot \boldsymbol{e}_{\boldsymbol{q}, p}\right)^{2} C^{2}(K)}{\left(1-\exp \left\{-\beta \omega_{\boldsymbol{q}, p}\right\}\right)\left(\exp \left\{\beta \omega_{\boldsymbol{q}, p}\right\}-1\right)} \frac{\mathrm{d} S \mathrm{~d} S^{\prime}}{v} v^{\prime}$

$W=\frac{9 \pi \hbar}{2 e^{2} L_{0} M N k_{\mathrm{B}} T^{2} k_{\mathrm{F}}{ }^{2} S_{\mathrm{F}}{ }^{2}} \sum_{p} \iint\left(\boldsymbol{K} \cdot \boldsymbol{e}_{\boldsymbol{q}, p}\right)^{2} C^{2}(K)$

$$
\times \frac{\left(\frac{K^{2}}{3}-\frac{K^{2}}{6 \pi^{2}}\left(\beta \omega_{\boldsymbol{q}, p}\right)^{2}+\frac{k_{\mathrm{F}}^{2}}{\pi^{2}}\left(\beta \omega_{\boldsymbol{q}, p}\right)^{2}\right)}{\left(1-\exp \left\{-\beta \omega_{\boldsymbol{q}, p}\right\}\right)\left(\exp \left\{\beta \omega_{\boldsymbol{q}, p}\right\}-1\right)} \frac{\mathrm{d} S S^{\prime}}{v} \frac{(2}{v^{\prime}} .
$$

Here $C(K)$ is the matrix element for the transition of an electron from initial to finite state on the Fermi surface, $v$ the electron velocity in state $\boldsymbol{k}$ on

5 R. P. Gupta and P. K. Sharma, J. Chem. Phys. 46, 1359 [1967]. - P. K. Sharma and K. N. Mehrotra, Anales Fis. 65, 189 [1969]. - P. K. Sharma and R. P. Gupta, Z. Physik. Chem. 242, 341 [1969].

6 M. Kohler, Z. Physik 124, 772 [1948] ; 125, 679 [1949].

7 E. H. Sondheimer, Proc. Roy. Soc. London A 203, 75 [1950]. 
the Fermi surface, $k_{\mathrm{F}}$ the Fermi momentum, $\boldsymbol{K}$ the scattering vector, $\omega_{\boldsymbol{q} . p}$ the angular frequency of a phonon of wave vector $\boldsymbol{q}$ and polarization $p, \boldsymbol{e}_{\boldsymbol{q}}, p$ the polarization vector, $M$ the ion mass, $N$ the number of ions per unit volume, $L_{0}$ the free electron Lorentz number, $T$ the absolute temperature, $k_{\mathrm{B}}$ the Boltzmann constant and $\beta$ is equal to $\hbar / k_{\mathrm{B}} T$. The two surface integrals are taken over the Fermi surface. Even for a spherical geometry, the evaluation of the double surface integral presents great difficulties because of the change of phonon parameters with the direction of $\boldsymbol{K}$. Here we use BAILYN's ${ }^{8}$ averaging procedure to convert the four-dimensional integral into a three-dimensional integral over $\boldsymbol{K}$. The details of the procedure have been described elsewhere ${ }^{9}$. Using this method, Eqs. (1) and (2) can be written as

$$
\begin{aligned}
\varrho= & \frac{3 \hbar}{4 \pi e^{2} M N k_{\mathrm{B}} T v_{\mathrm{F}}^{2} k_{\mathrm{F}}^{3}} \sum_{p} \int \mathrm{d} \Omega \int \mathrm{d} K \\
& \times \frac{K^{2}\left(1-u^{2}\right)^{1 / 2}\left(\mathbf{K} \cdot \boldsymbol{e}_{\boldsymbol{q}, p}\right)^{2} C^{2}(K)}{\left(1-\exp \left\{-\beta \omega_{\boldsymbol{q}, p}\right\}\right)\left(\exp \left\{\beta \omega_{\boldsymbol{q}, p}\right\}-1\right)},
\end{aligned}
$$

Here $\Omega$ is the solid angle in $\boldsymbol{K}$ space and $u=K / 2 k_{\mathrm{F}}$. It is obvious from Eqs. (3) and (4) that to evaluate $\varrho$ and $W$ we need to know the matrix element $C(K)$ and the phonon dispersion relations. In the present work we have used the free electron model for $C(K)$ from ZimaN ${ }^{1}$, and SHARMA and Joshi's model ${ }^{4}$ for the phonon dispersion relation $\omega_{\boldsymbol{q}, p}$.

\section{Results and Discussion}

The evaluation of electrical and thermal resistivities of copper, silver and gold at different temperatures from Eqs. (3) and (4) has been made by a modification of Houston's interpolation procedure ${ }^{\mathbf{1 0}}$. The integration over $\boldsymbol{K}$ was performed numerically and over $\Omega$ by the six-term integration method as developed by BETTS et al. ${ }^{11}$. The six directions for $\mathbf{K}$ used are: [100], [110], [111], [210], [211] and [221]. The phonon frequencies and polarization vectors were obtained from solutions of the SharmaJoshi secular determinant ${ }^{4}$ for a face-centred cubic lattice. Two sets of elastic constants were used referring to $0 \mathrm{~K}$ and $300 \mathrm{~K}$. The numerical values of elastic constants and other parameters used in the calculation are given in Table 1 . The elastic constants have been taken from the measurements of Overton and GAFFNEY ${ }^{12}$ for copper, and from Neighbours and Alers ${ }^{13}$ for silver and gold. In evaluating the integrals over $K$ in Eqs. (3) and (4), the normal and Umklapp processes were considered

\begin{tabular}{|c|c|c|c|c|c|c|c|}
\hline Metal & $C_{11}$ & $\begin{array}{c}\text { Elastic constants } \\
\left(10^{11} \text { dynes } / \mathrm{cm}^{2}\right) \\
C_{12}\end{array}$ & $C_{44}$ & $\begin{array}{l}\text { Lattice } \\
\text { parameter } \\
(\AA)\end{array}$ & $\begin{array}{c}\text { Temperature } \\
\text { (K) }\end{array}$ & $\begin{array}{c}V\left(r_{\mathrm{s}}\right)-E_{0} \\
(\mathrm{eV})\end{array}$ & $\begin{array}{c}\text { Fermi energy } \\
(\mathrm{eV})\end{array}$ \\
\hline Copper & $\begin{array}{l}17.620 \\
16.839\end{array}$ & $\begin{array}{l}12.494 \\
12.142\end{array}$ & $\begin{array}{l}8.177 \\
7.539\end{array}$ & $\begin{array}{l}3.604 \\
3.616\end{array}$ & $\begin{array}{c}0 \\
300\end{array}$ & $\begin{array}{l}1.361 \\
1.361\end{array}$ & $\begin{array}{l}7.04 \\
7.04\end{array}$ \\
\hline Silver & $\begin{array}{l}13.149 \\
12.399\end{array}$ & $\begin{array}{l}9.733 \\
9.367\end{array}$ & $\begin{array}{l}4.109 \\
4.612\end{array}$ & $\begin{array}{l}4.078 \\
4.080\end{array}$ & $\begin{array}{c}0 \\
300\end{array}$ & $\begin{array}{l}1.10 \\
1.10\end{array}$ & $\begin{array}{l}5.51 \\
5.51\end{array}$ \\
\hline Gold & $\begin{array}{l}20.163 \\
19.234\end{array}$ & $\begin{array}{l}16.967 \\
16.314\end{array}$ & $\begin{array}{l}4.544 \\
4.195\end{array}$ & $\begin{array}{l}4.054 \\
4.070\end{array}$ & $\begin{array}{c}0 \\
300\end{array}$ & $\begin{array}{l}3.70 \\
3.70\end{array}$ & $\begin{array}{l}5.51 \\
5.51\end{array}$ \\
\hline
\end{tabular}

Table 1. Constants for noble metals used in the calculation.

8 M. Bailyn, Phys. Rev. 120, 381 [1960].

9 P. K. Sharma and R. P. Gupta, Rev. Mex. Fis. 18, 41 [1969].

10 A. A. Maradudin, E. W. Montroll, and G. H. Weiss, Theory of Lattice Dynamics in Harmonic Approximation, Academic Press, Inc., New York 1963.
11 D. D. Betts, A. B. Bhatia, and M. Wyman, Phys. Rev. 104, 37 [1956].

12 W. C. Overton and J. Gaffney, Phys. Rev. 98, 969 [1955].

13 J. R. Neighbours and G. A. Alers, Phys. Rev. 111, 707 [1958]. 
separately from the conservation laws of wave vectors and the geometry of reciprocal space. For normal processes, $\boldsymbol{K}=\boldsymbol{q}$, where the phonon wave vector $\boldsymbol{q}$ lies within the first Brillouin zone. The limiting values of $\boldsymbol{K}$ along any direction are given by the intersection of corresponding $\boldsymbol{K}$ vectors with the planes of the first Brillouin zone. In Umklapp processes $\boldsymbol{K}=\boldsymbol{q}+\boldsymbol{G}$, where $\boldsymbol{G}$ is a vector of the reciprocal space. In this case $\boldsymbol{K}$ goes beyond the boundary of the first Brillouin zone, but the phonon wave vector is constraint to lie inside the Brillouin zone. The minimum values of $\boldsymbol{K}$ along the above six directions at which Umklapp processes start up were obtained from the geometry of the reciprocal space.

Figures 1 and 2 show the calculated electrical and thermal resistivities of noble metals at different temperatures. For comparison the experimental values have also been plotted in these figure. The sources of experimental resistivity data are summarized in Table 2. The experimental resistivity values reported by various authors agree well enough excep! for copper in the low temperature region. It will be

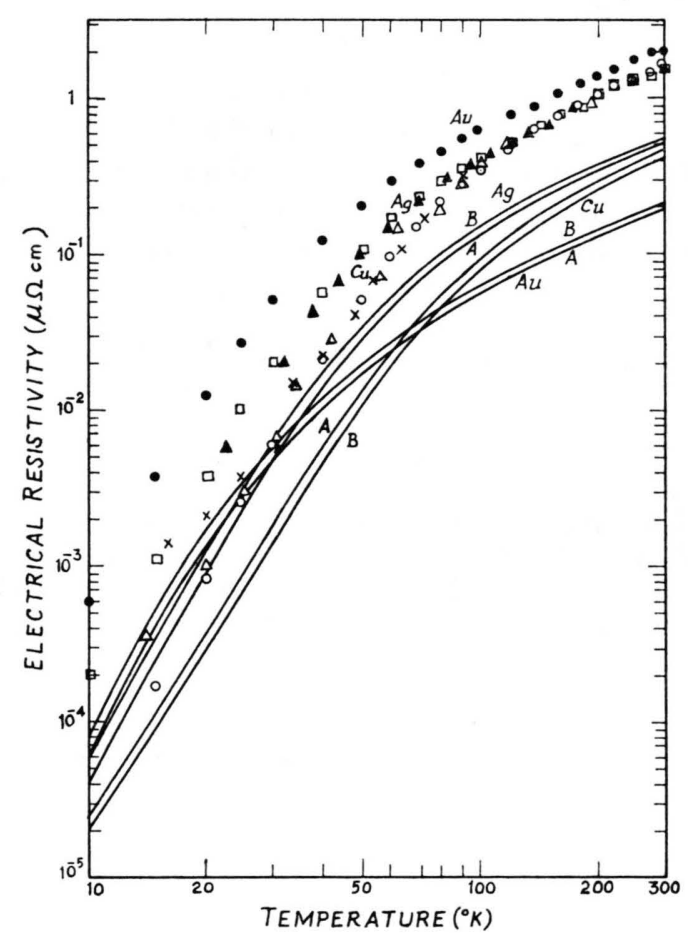

Fig. 1. The electrical resistivity against temperature for noble metals. The curves A and B are obtained by using elastic data at $0 \mathrm{~K}$ and $300 \mathrm{~K}$ respectively. Experimental points, $\mathrm{Cu}: \bigcirc$, White and Woods, $X$, Berman and MacDonald, $\triangle$, White;

Ag: $\square$, White and Woods, $\boldsymbol{\Delta}$, Dugdale and Basinski; Au: White and Woods.

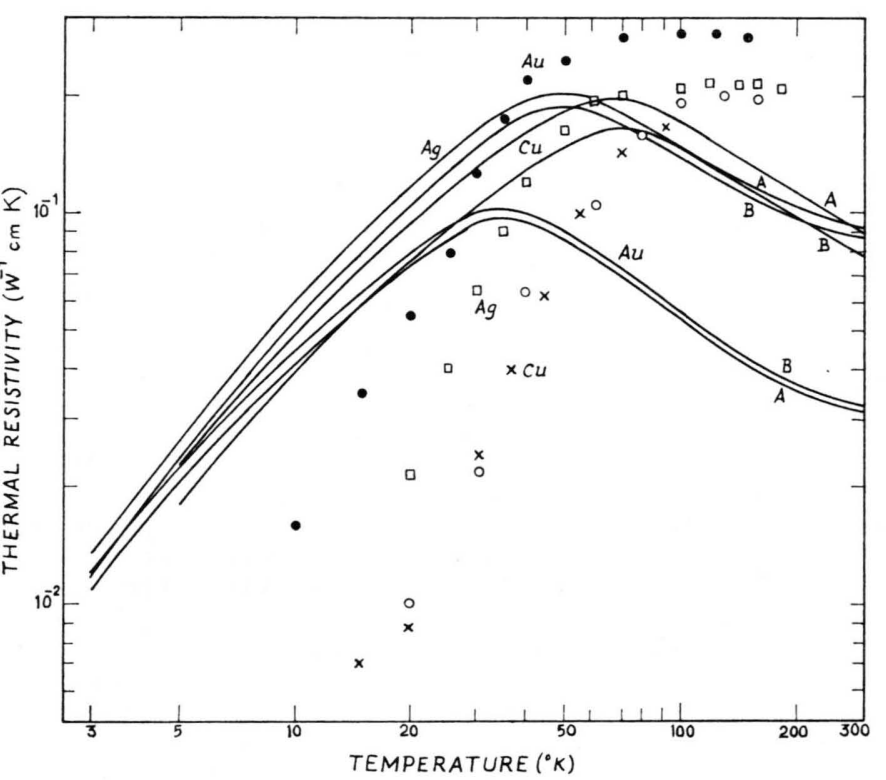

Fig. 2. The thermal resistivity versus temperature curve for noble metals. The curves A and B correspond to elastic data at $0 \mathrm{~K}$ and $300 \mathrm{~K}$ respectively. Experimental points, $\mathrm{Cu}: \times$, Berman and MacDonald, $\bigcirc$, White; Ag: $\square$, White; Au: White.

seen from Fig. 1 that the nature of the theoretical and experimental resistivity curves is similar, but the experimental values are higher than the theoretical ones throughout the temperature range studied and the discrepancy increases with rising temperature. Figure 2 shows that the shape of the theoretical thermal resistivity curves is quite right, but the agreement with experiments is not satisfactory at low and high temperatures. At low temperatures the calculated values are substantially higher than the experimental values, while at high temperatures the theoretical curves lie below the experimental points.

The discrepancies between theory and experiment at low and high temperatures may be ascribed to the neglect of several effects. The expressions (1) and (2) are obtained on one-phonon approximation and first-order trial function in the variational solution of the Boltzmann equation. The first assumption is expected to be true at low temperatures, but the use of the first order variational solution somewhat overestimates the resistance. Further, the present study uses the free electron model for the electronphonon matrix element which overlooks the exchange and correlation effects. If the exchange and correlation energies are introduced in $C(K)$, the 
Table 2. Experimental electrical and thermal resistivity data for noble metals.

\begin{tabular}{|c|c|c|c|c|}
\hline \multirow[t]{2}{*}{ Metal } & \multicolumn{2}{|r|}{ Electrical resistivity } & \multicolumn{2}{|r|}{ Thermal resistivity } \\
\hline & $\begin{array}{l}\text { Temperature } \\
\text { range }(\mathrm{K})\end{array}$ & Source & $\begin{array}{l}\text { Temperature } \\
\text { range }(\mathrm{K})\end{array}$ & Source \\
\hline Copper & $\begin{array}{r}1.4-290 \\
2-90 \\
15-295\end{array}$ & $\begin{array}{l}\text { WHITE a } \\
\text { BERMAN and MACDONALD b } \\
\text { WHITE and WOODS c }\end{array}$ & $\begin{array}{l}2-90 \\
2-160\end{array}$ & $\begin{array}{l}\text { BERMAN and MacDonald b } \\
\text { WHITE a }\end{array}$ \\
\hline Silver & $\begin{array}{l}10-295 \\
22-298\end{array}$ & $\begin{array}{l}\text { WHITE and Woods c } \\
\text { DugDALE and BASINSKI d }\end{array}$ & $2-160$ & White e \\
\hline Cold & $10-295$ & WhITE andn Woods $\mathrm{c}$ & $2-150$ & WhITE $f$ \\
\hline
\end{tabular}

: G. K. White, Austr. J. Phys. 6, 397 [1953].

b. R. Berman and D. K. C. MacDonald, Proc. Roy. Soc. London A 211, 122 [1952].

c G. K. White and S. B. Woods, Phil. Trans. Roy. Soc. London A 251, 373 [1959].

'J J. S. Dugdale and Z. S. Basinski, unpublished (private communication).

- G. K. White, Proc. Phys. Soc. London A 66, 844 [1953].

f G. K. White, Proc. Phys. Soc. London A 66, 559 [1953].

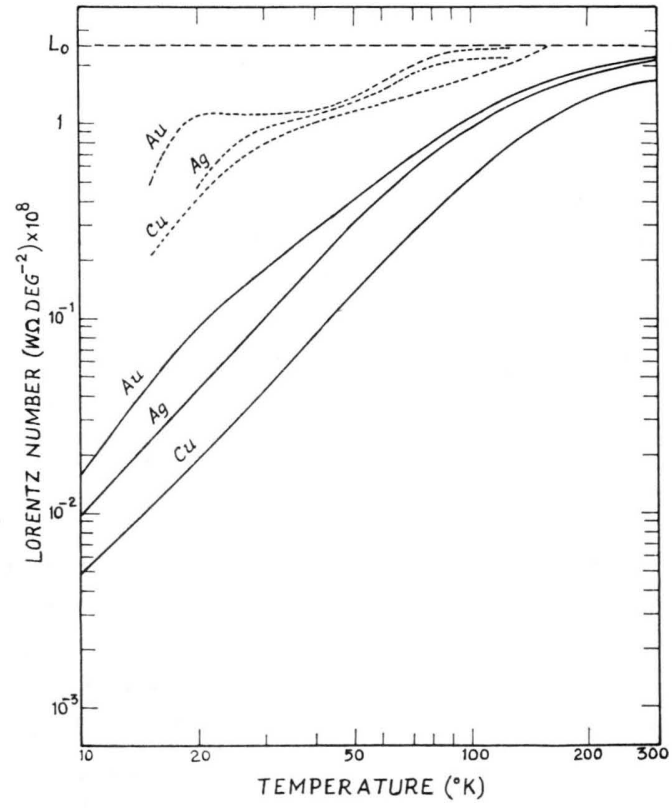

Fig. 3. The Lorentz number of noble metals. The solid curves represent present calculations and the dashed curves are obtained from experimental resistivity data. form factors are slightly increased. This will have a pronounced effect on the electrical resistivities at higher temperatures. At high temperatures multiphonon processes will also have a dominant effect on the transport coefficients.

Using the calculated temperature dependence of resistivities shown in Figs. 1 and 2, the Lorentz number $L=\varrho / T W$ of noble metals is determined. Figure 3 shows the calculated values of $L$ along with the curves deduced from experimental resistivity data. It is seen that the theoretical and experimental Lorentz numbers vary with temperature and show marked departure from the Wiedemann-Franz Law.

\section{Acknowledgements}

The authors are thankful to the Council of Scientific and Industrial Research, New Delhi, for financial help. The work was partly supported by the Department of Atomic Energy. 